\title{
Multiple Sagittal Sinus Arteriovenous Fistula Resulting from a Transverse Sinus Venous Thrombosis Presenting with Superior Orbital Vein Engorgement and Status Epilepticus: A Case Report
}

\author{
Mari Anne Kristin Dale N. Moll, Patricia Ann D. Canto, \\ Leah L. Shiong Shu and Jose Leonard R. Pascual \\ Department of Neurosciences, College of Medicine and Philippine General Hospital, University of the Philippines Manila
}

\begin{abstract}
A 45-year-old female presented with a 7-year history of right superior orbital vein engorgement, later developing status epilepticus. Cerebral catheter angiogram done revealed 13 dural arteriovenous fistula (DAVFs), two pial arteriovenous fistula, an occluded right transverse sinus and a markedly congested right superior ophthalmic vein.
\end{abstract}

DAVFs are very rare lesions. As of this writing,there has been no published local data on DAVFs presenting as such. This is the first reported case.

Key Words: dural/ pial arteriovenous fistula, venous hypertension, venous thrombosis, arteriorvenous shunting

\section{Introduction}

Dural arteriovenous fistulas (DAVF) are very uncommon vascular abnormalities whose incidence and pathogenesis are not completely known. They constitute 10$15 \%$ of all intracranial arteriovenous shunts, ${ }^{1}$ however, because this condition can be clinically silent, the true incidence remains unknown. The reported annual morbidity and mortality rates of DAVFs with an aggressive presentation vary broadly, ranging from $1.8 \%$ to $20 \%$ per year. ${ }^{2}$

DAVFs are arteriovenous shunts between abnormal arteries and veins contained within the dura. They may be congenital or acquired. The exact mechanism of their formation has not been well established; however, they are believed to be generally acquired most commonly from venous thrombosis.
Corresponding author: Mari Anne Kristin Dale N. Moll, MD Department of Neurosciences

Philippine General Hospital

University of the Philippines Manila

Taft Avenue, Ermita, Manila 1000 Philippines

Telefax No.: +632 5548462

Email: dale_moll@yahoo.com

\section{Case Presentation}

This is the case of a 45-year-old, right-handed, female Filipino who had been hypertensive for 3 years with poor compliance to medications. She presented with a 7-year history of a gradually enlarging superior orbital vein over the right upper eyelid with associated bruit and redness of her right eye (Figure 1). There was no history of trauma, no complaints of pruritus, ophthalmoplegia or proptosis. On consult with an ophthalmologist 5 years prior to admission, initial assessment was a possible carotico-cavernous fistula. She was advised to have an angiogram but was not able to have the procedure done due to financial difficulties.

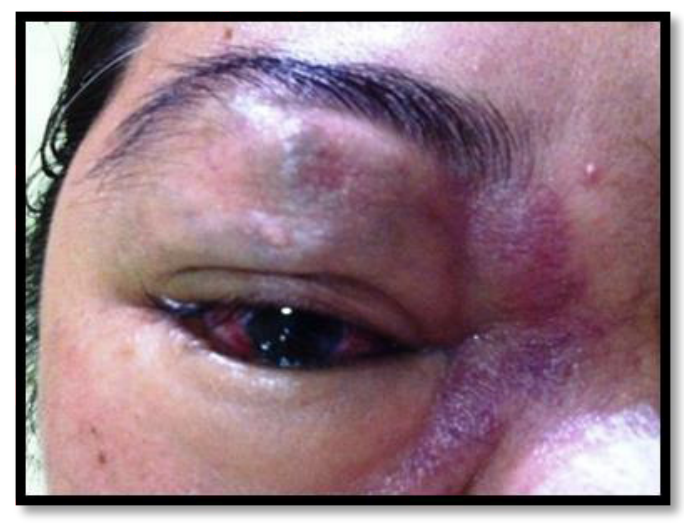

Figure 1. Vessel engorgement on the right upper eyelid

Two months prior to admission, the patient started having intermittent memory lapses during which she could not perform simple tasks such as buying necessities at the store because she could not remember the items on her list.

On the day of admission, the patient was seen confused in the bathroom. She began having focal seizures with secondary generalization occurring in clusters. She was brought immediately to a local hospital and was given diazepam.

She arrived at the emergency room in status epilepticus and was intubated. Remarkable in the physical examination were the presence of an engorged orbital vein on the right upper eyelid with bruit, palpable thrills, and injection of the right eye. She was stuporous with eye opening to pain, had 
reactive and equal pupils, intact Doll's eye, no facial asymmetry, no auditopalpebral reflex, intact gag, able to withdraw to pain, hyperreflexic lower extremities, no extensor toe sign, and supple neck.

Cranial CT Scan revealed multiple calcifications found bilaterally over the frontal lobes with dilated transverse sinuses, as well as cortical vein thrombosis over the left parietal and right temporal areas (Figure 2). On contrast cranial CT Scan, there were multiple serpigionous enhancement and venous dilatations (Figure 3). The scan warranted a cerebral catheter angiogram for further investigation.

A cerebral catheter angiogram showed a total of 13 dural areteriovenous fistula (DAVFs) and two pial AVFs scattered on both hemispheres, and both supratentorial and intratentorial.

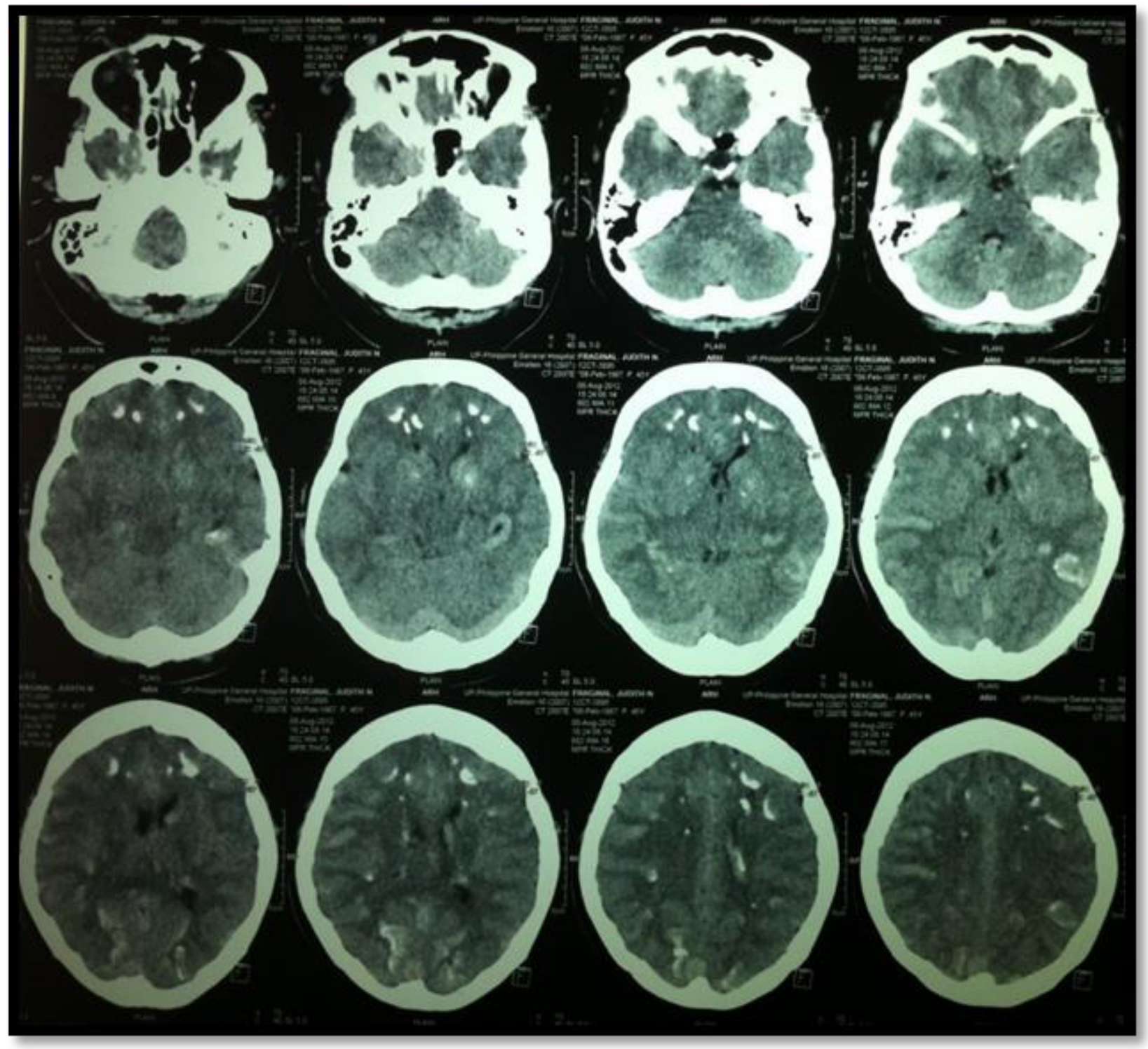

Figure 2. Plain cranial CT scan 


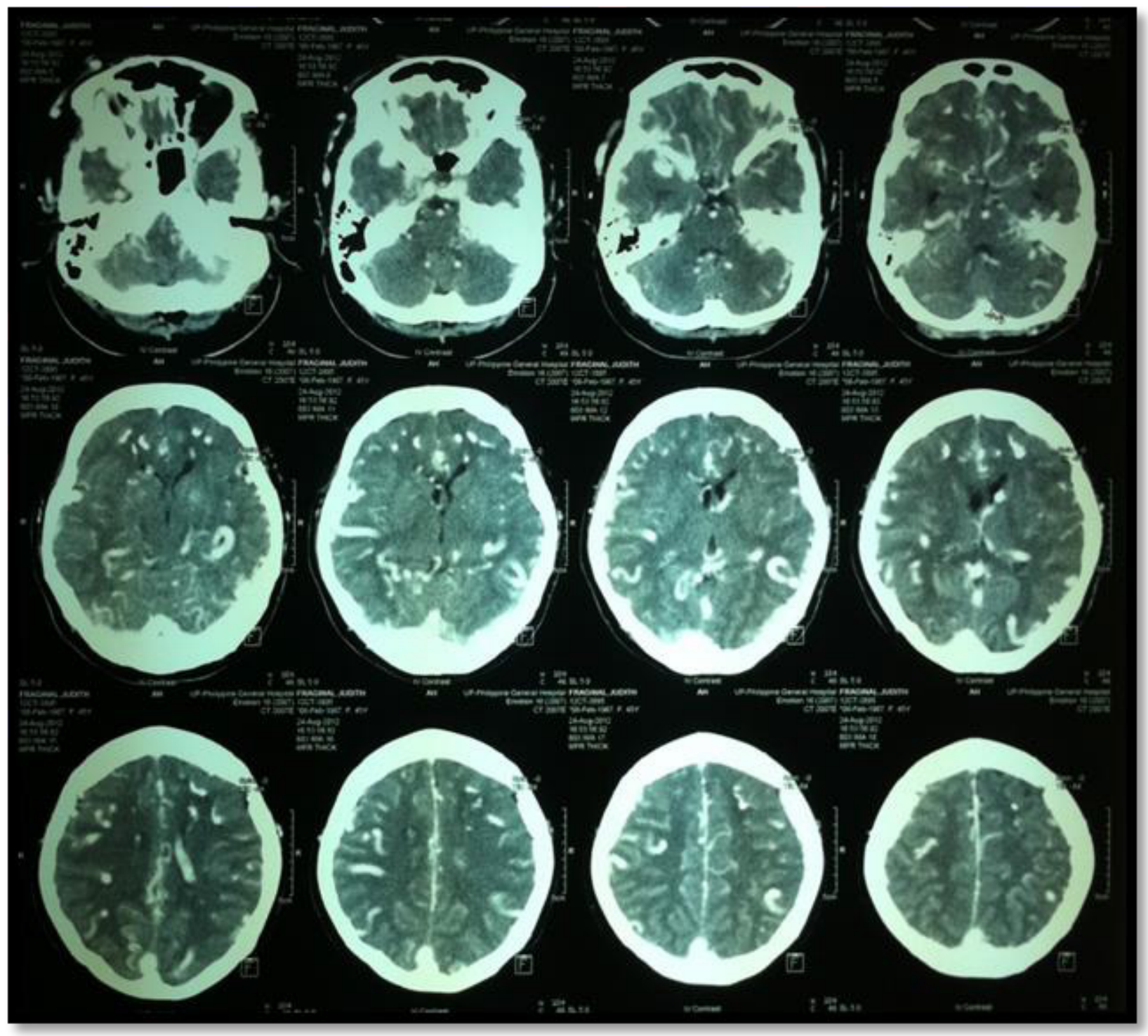

Figure 3. Cranial CT Scan with contrast

The internal carotid angiogram (Figures 4A and 4B) revealed DAVFs emptying into the anterior and middle portion of the Superior Sagittal Sinus (SSS) and involvement of frontal cortical veins. There were also two pial AVFs noted.

Left vertebral angiogram (Figure 4C) revealed DAVF formation between the left vertebral artery and cerebellar cortical veins.

The external carotid angiogram (Figures 4D and 4E) showed DAVF formation between the occipital artery and the posterior portion of the SSS.

Aside from the multiple arteriovenous fistula seen, venous phase showed occlusion of the distal half of the right transverse sinus. There were venous collaterals via the superior petrosal- right cavernous sinus with reflux into a markedly dilated right superior ophthalmic and angular veins. There also appeared to be an occlusion of the right interior petrosal sinus. Due to the arterialization of the superior petrosal and transverse sinuses, there was reflux into the cortical vein tributaries of these sinuses.

There was no evident carotico-cavernous fistula but a dilated right superior ophthalmic vein was appreciated is due to the venous collateral outflow from the occluded distal right transverse sinus.

Treatment options offered included embolization and resection of the superior sagittal sinus. However, the patient's family opted to have conservative and supportive management. Medical decompression was performed and anticonvulsants were started. She was lifted from status epilepticus and was discharged improved on close follow up at the outpatient department. 

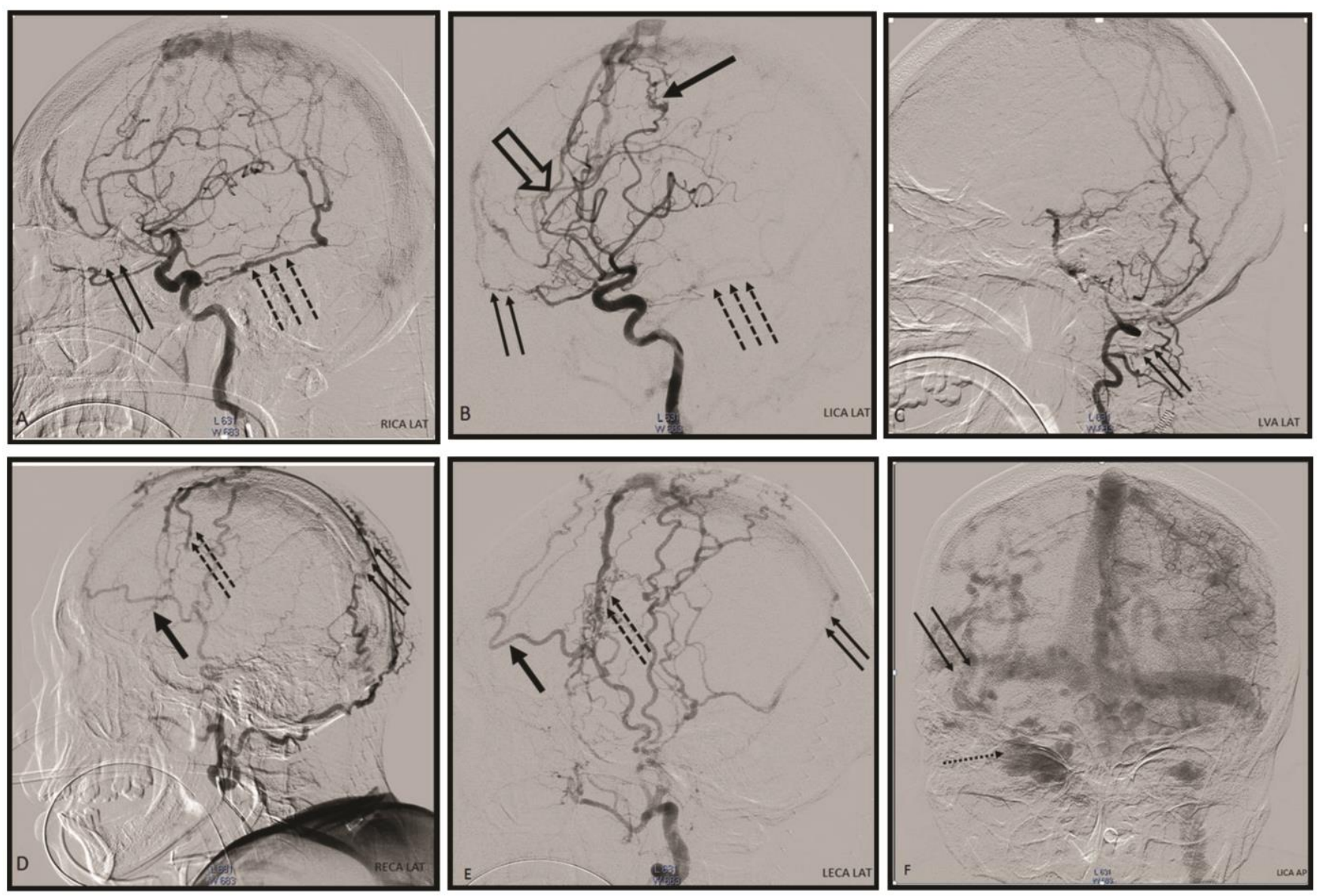

Figure 4. A. Right internal carotid angiogram, dashed arrows show DAVF between the dorsal tentorial branch of the right meningohypophyseal trunk and the tentorial veins which empty into the anterior and middle portion of the superior sagittal sinus. Thin arrows show DAVF between the falx cerebri artery arising from the right ophthalmic artery and the frontal corical veins which empty into the anterior portion of the superior sagittal sinus. B. Left internal carotid angiogram, dashed arrows show DAVF between the dorsal tentorial branch of the left meningohypophyseal trunk and the tentorial veins which empty into the anterior and middle portion of the superior sagittal sinus. Thin arrows show DAVF between the falx cerebri artery arising from the left ophthalmic artery and the frontal corical veins which empty into the anterior portion of the superior sagittal sinus. The thick arrow shows DAVF between the central branch of the left middle cerebral artery and central portion of the superior sagittal sinus. The hollow arrow shows the posterior internal frontal branch of the left callosomarginal artery forming a DAVF with the anterior portion of the superior sagittal sinus. C. Left vertebral angiogram, lateral view: thin arrows show fistulae ormation between the muscular branches of the left vertebral artery with the cerebellar cortical veins which empties towards the anterior parietal portion of the SSS. D. Right external carotid angiogram, lateral view; thick arrow shows the DAVF between right superficial temporal artery and anterior SSS, dashed arrows show DAVF between right middle meningeal artery and middle part of the SSS, thin arrow shows the DAVF draining into the posterior portion of the SSS from the occipital artery. E. Left external carotid angiogram, lateral view; thick arrow shows the DAVF between left superficial temporal artery and anterior SSS, dashed arrow shows DAVF between left middle meningeal artery and middle part of the SSS, thin arrow shows the DAVF draining into the posterior portion of the SSS from the left posterior auricular and left occipital arteries. F. Venous phase: thin arrows show occlusion of the distal half of the right transverse sinus, dashed arrow shows venous collaterals via the superior petrosal-right cavernous sinus with reflux into a markedly dilated right superior ophthalmic and angular veins. 


\section{Discussion}

Dural arteriovenous fistulas (DAVF) are very rare vascular abnormalities whose nature is not yet fully understood. They constitute $10-15 \%$ of all intracranial arteriovenous shunts; ${ }^{1}$ however, because the condition may be clinically silent, the true incidence remains unknown. According to a report by Singh, dural AVFs has an incidence of 0.17 per 100,000 persons. ${ }^{3}$ The reported annual morbidity and mortality rates of DAVFs with an aggressive presentation vary broadly, ranging from $1.8 \%$ to $20 \%$ per year. $^{2}$ Very little is known regarding the true nature of DAVFs.

There is a diverse range of hypotheses on the formation of dural AVFs, whether they are congenital or acquired. However, they are generally considered acquired most especially when they present at a later stage in life such as in the case of our patient. Many etiologic factors have also been associated with the formation of dural AVFs. Infection, trauma, and previous brain surgery are a few of these. Still, the best-defined examples of acquired fistulas are those that arise adjacent to a venous sinus thrombosis or in association with a vascular atresia, most often of the transverse sigmoid sinus or adjacent to the cavernous sinus. ${ }^{4}$ Our patient had venous thrombosis of the right transverse sinus. In this case, we would expect DAVFs to form adjacent to this thrombosed sinus; however, the patient's DAVFs were seen in various areas of the brain, draining into the SSS.

Kusaka reported the case of a 55-year-old man who developed multiple fistulas, including one on the cavernous sinus, which were remote from sinus thrombosis seen on the left transverse sinus. Here he implicated venous hypertension in the development DAVFs. ${ }^{5}$ Venous thrombosis promotes venous hypertension, which acts as the initiating factor opening up microscopic vascular connections within the dura. Maturation of these channels secondary to progressive venous stenosis or occlusion results in the development of direct shunts between the arteries and dural veins. There could have been a longstanding right transverse venous thrombosis in our patient sufficienct to promote venous hypertension, which explains the multiplicity and scattered distribution of her DAVFs.

Our patient also has two pial AVFs. Intracranial pial AVFs are also rare cerebrovascular lesions, with less than 100 reported cases since 1970. Pial AVFs differ from DAVFs in that the arterial supply is derived from pial and cortical arterial vessels and the location is not within the dural leaflets. ${ }^{6}$ Like DAVFs, not much is known regarding the pathophysiology of pial AVFs. However, unlike DAVFs, pial AVFs are believed to be congenital. It has been suggested that a defect in the embryological development of the cerebrovasculature may contribute to their formation; however, an acquired etiology is not completely excluded. A report by Phataoros described the unusual case of a 51-yearold man with acquired pial AVFs which occurred after a cortical vein thrombosis. ${ }^{7}$ Venous hypertension may still contribute to the formation of pial AVFs.

One of the main obstacles in appreciating DAVFs is the diversity of their manifestations. Most case reports account for patients presenting with one of the following symptoms: seizures, eye signs such as proptosis and chemosis or bruit and tinnitus. The most dreaded presentation is intracranial hemorrhage, whether subdural, subarachnoid or intraparenchymal. Studies have shown that symptoms depend primarily on the location of the DAVFs. This case report is unique in that the DAVFs had numerous manifestations. The patient had ophthalmic vein engorgement, injection of the right eye, tinnitus, cognitive decline, status epilepticus, and intracranial hemorrhage, all owing to the multiplicity of her lesions.

Sencer and Kiris ${ }^{8}$ classified DAVFs according to location. Transverse-sigmoid sinus DAVFs make up the majority of DAVFs. Cavernous sinus DAVFs (10-16\%), tentorial DAVFs (8-12\%), superior sagittal sinus DAVFs $(8 \%)$, and anterior cranial fossa DAVFs (5\%) and rare locations (torcula, foramen magnum, deep venous fistula) are other types of DAVFs. In a study by Choi et al., ${ }^{9}$ the authors reported that the transverse-sigmoid sinus is the most frequent site of occurrence in Western countries, while the cavernous sinus is the most common location of DAVF in Asians.

Our patient presented with seizures that could be explained by the cortical lesions as well as regional hypoxia caused by the DAVFs, also known as the steal phenomenon. In the same paper by Sencer and Kiris, the authors reported that SSS DAVFs usually present with intracranial bleeding and dementia. ${ }^{8}$ Our patients' ophthalmic vein engorgement which was initially thought to be a carotico-cavernous fistula may also suggest that the right transverse sinus occlusion has been present long enough to cause venous reflux into the right superior ophthalmic and angular veins.

The Borden Classification system is used to classify DAVFs and has significant correlation to clinical presentation. Type I DAVFs drain into a dural venous sinus or meningeal vein with normal anterograde flow. These usually have benign clinical behavior. Type II DAVFs have anterograde drainage into dural venous sinus and onwards but retrograde flow occurs into cortical veins. These may present with hemorrhage. Type III DAVFs have direct retrograde flow of blood from the fistula into cortical veins causing venous hypertension with a risk of hemorrhage. ${ }^{10}$

Our patient is classified Borden Type III. According to Soderman et al., the risk of intracranial hemorrhage from a dural arteriovenous shunt with cortical venous drainage is most likely smaller to what has been previously proposed. ${ }^{11}$ The authors followed five patients with Borden type III until death. Four of the patients refused any treatment or radiological follow up after diagnostic angiography. Only one died of a deep frontal-parietal bleed. The rest died of 
other causes: ruptured aortic aneurysm, alcohol abuse, trauma, and congestive heart failure. The annual incidence of intracranial hemorrhage and mortality is thus $1.7 \%$. This does not support the hypothesis of a high risk of hemorrhage from DAVFs. ${ }^{11}$

In a study by Davies et al. on 46 patients with Borden II and III DAVFs, 14 patients received conservative management, 22 received surgery, and 20 received embolization. In the conservative group, that patients had interval rates of intracranial hemorrhage, non-hemorrhagic neurological deficit, and mortality of $19.2 \%, 10.9 \%$, and $19.3 \%$ per lesion year, respectively. ${ }^{12}$

Treatment is dependent on the clinical picture and the grade of fistula. Though in all DAVFs, it is prudent to pursue treatment, a multidisciplinary approach involving a neurologist, neurosurgeon and neuroradiologist is required to weigh the risks of treatment against the natural course of DAVFs.

Both surgical and endovascular techniques have proven efficacy for more troublesome DAVFs. ${ }^{10}$ In endovascular therapy, which is the first-line treatment for DAVFs, treatment is aimed at complete elimination of the arteriovenous shunt because incomplete treatment allows recruitment of collateral vessels and persistent risk of hemorrhage. However, in cases where endovascular therapy is not feasible or has failed, surgical disconnection of the cortical venous drainage may be performed. A variety of options is available, including direct intraoperative embolization of meningeal arteries or veins, resection of abnormal dura, packing of the diseased sinus, disconnection of the retrograde leptomeningeal venous drainage, and skeletonization of the dural sinus with disconnection of the dural arterial supply. ${ }^{13}$

Stereotactic radiosurgery (SRS) has also showed promising results when it comes to the treatment of DAVFs. In a study by Gross et al., treatment of 9 cases of DAVFs with SRS obtained an overall obliteration rate of $71 \%$, which was observed to be greatest in patients with cavernous DAVFs and those without cortical venous drainage. ${ }^{14}$

For this patient, embolization or surgical disconnection of the arterialized draining vein may be employed with close follow up and monitoring of the development of new arteriovenous shunts.

\section{Conclusion}

We presented a very rare case of multiple superior sagittal sinus DAVFs and pial AVFs believed to be acquired from a possible long-standing transverse sinus thrombosis. This case is particularly unique in that the said lesions formed remote from the thrombosed sinus attributable to venous hypertension.

As of this writing there has been no published local data on DAVFs presenting as such: this is the first reported case.
With this case, we learned the importance of considering dural AVFs as a differential diagnosis in patients presenting with any vascular malformations within the cranial vault, most especially in the orbit and that further investigation for other causes of vascular diseases is warranted for such cases so that appropriate management may be rendered and complications are prevented.

\section{References}

1. Radiology, Involvement of dural arteries in intracranial arteriovenous malformations. [Online]. 1969 [cited 2012 Aug]. Available from http://radiology.rsna.org/content/93/5/1071.extract?relatedurls=yes\&legid=radiology; $93 / 5 / 1071$

2. Van Dijk JM, terBrugge KG, Willinsky RA, Wallace MC. Clinical course of cranial dural arteriovenous fistulas with long-term persistent cortical venous reflux. Stroke. 2002; 33(5):1233-6.

3. Singh RK. Dural AVF - Classification and Management [Online]. [cited 2012 Aug]. Available from http://aiimsnets.org/NeurosurgeryEducation/ NeurosurgicalSpecialties/Skullbaseandvascularneurosurgery/duralAVF/ DURALAVF.pdf.

4. Ropper AH, Samuels MA. Adams and Victor's Principles of Neurology, 9th ed. United States: The McGraw-Hill Companies, Inc.; 2009. pp 819820.

5. Kusaka N, Sugiu K, Katsumata A, Nakashima H, Tamiya T, Ohmoto T. The importance of venous hypertension in the formation of dural arteriovenous fistulas: a case report of multiple fistulas remote from sinus thrombosis. Neuroradiology. 2001; 43(11):980-4.

6. Yamashita $\mathrm{K}$, Ohe $\mathrm{N}$, Yoshimura $\mathrm{S}$, Iwama $\mathrm{T}$. Intracranial pial arteriovenous fistula. Neurol Med Chir (Tokyo). 2007; 47(12):550-4.

7. Phatouros CC, Halbach VV, Dowd CF, et. al. Acquired pial arteriovenous fistula following cerebral vein thrombosis. Stroke. 1999; 30(11):2487-90.

8. Sencer A, Kiris T. Intracranial dural arteriovenous fistulas: a brief review on classification and general features. Turk Neurosurgery. 2006; 16(2):57-64.

9. Choi BS, Park JW, Kim JL, et al. Treatment strategy based on multimodal management outcome of Cavernous Sinus Dural Arteriovenous Fistula (CSDAVF). Neurointervention. 2011; 6(1):6-12.

10. Adams W, Whitfield P. Intracranial dural arteriovenous fistulae. Adv Clin Neurosci Rehab. 2007; 7(3):10-2.

11. Söderman M, Pavic L, Edner G, Holmin S, Andersson T. Natural history of dural arteriovenous shunts. Stroke. 2008; 39(6):1735-9.

12. Davies MA, Ter Brugge K, Willinsky R, Wallace MC. The natural history and management of intracranial dural arteriovenous fistula Part 2: aggressive lesions. Interv Neuroradiol. 1997; 3(4):303-11.

13. Gandhi D, Chen J, Pearl M, Huang J, Gemmete JJ, Kathuria S. Intracranial dural arteriovenous fistulas: classification, imaging findings, and treatment. AJNR Am J Neuroradiol. 2012; 33(6):1007-13.

14. Gross BA, Ropper AE, Popp AJ, Du R. Stereotactic radiosurgery for cerebral dural arteriovenous fistulas. Neurosurg Focus. 2012; 32(5):E18. 


\section{APAME 2015 CONVENTION}

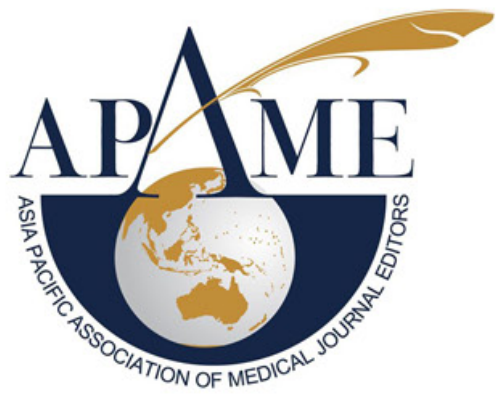

SAVE THE DATE! 24-26, AUGUST 2015

SOFITEL PHILIPPINE PLAZA HOTEL

PHILIPPINE INTERNATIONAL CONVENTION CENTER

PASAY CITY, METRO MANILA, PHILIPPINES

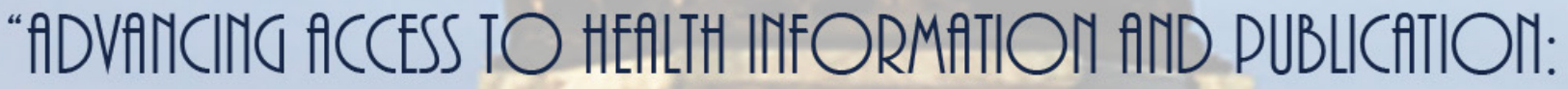 SHIFTIIIG DARADIIMSS, TREMDS AMID IMIIOVATIONI"}

http://apame2015.healthresearch.ph

HOSTED BY:

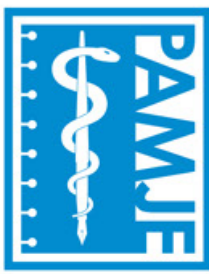

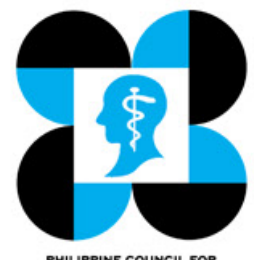
PHILIPPINE COUNCIL FOR
HEALTH RESEARCH AND DEVELOPMENT

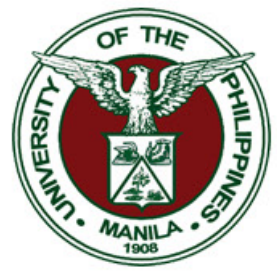

IN COOPERATION WITH:
IN CONJUNCTION WITH:

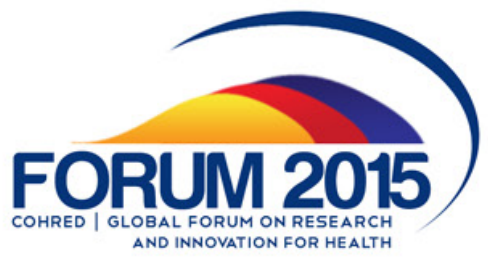

